\title{
Timing-Dependent Modulation of Associative Plasticity by General Network Excitability in the Human Motor Cortex
}

\author{
Michael A. Nitsche, Amelie Roth, Min-Fang Kuo, Anja K. Fischer, David Liebetanz, Nicolas Lang, Frithjof Tergau, and \\ Walter Paulus \\ Georg-August-University, Department for Clinical Neurophysiology, 37075 Goettingen, Germany
}

\begin{abstract}
Associative neuroplasticity, which encompasses the modification of synaptic strength by coactivation of two synaptic inputs, has been linked to learning processes. Because unlimited plasticity destabilizes neuronal networks, homeostatic rules were proposed and experimentally proven that control for the amount and direction of plasticity dependent on background network activity. Accordingly, low background activity would enhance facilitatory plasticity, whereas high background activity would inhibit it.

However, the impact of background excitability on associative plasticity has not been studied so far in humans. Facilitatory associative plasticity was induced by paired associative stimulation (PAS) in the human motor cortex, whereas background activity was enhanced or diminished by transcranial direct current stimulation (tDCS). When applied before PAS, excitability-enhancing tDCS also boosted the efficacy of PAS, whereas excitability-diminishing tDCS turned it into inhibition. Thus, previous background activity does not influence associative plasticity homeostatically. When tDCS and PAS were applied simultaneously, now in accordance with homeostatic rules of neuroplasticity, reduced background activity resulted in a prolonged excitability enhancement by PAS, whereas enhanced background activity turned it into inhibition. We conclude that background network activity can influence associative plasticity homeostatically. However, only simultaneous modulation of both parameters is in accordance with homeostatic concepts. These findings might be of importance for the development of plasticity-inducing stimulation protocols supporting information processing in humans.
\end{abstract}

Key words: homeostatic plasticity; paired associative stimulation; tDCS; TMS; motor cortex; human

\section{Introduction}

Understanding the neurophysiological basis of learning is an important endeavor of cognitive neuroscience research. Mechanisms, like long-term potentiation, may be involved (RioultPedotti et al., 1998, 2000; Ziemann et al., 2004; Stefan et al., 2006). Because unlimited neuroplasticity would destabilize neuronal networks, homeostatic mechanisms have been proposed to control for balanced network modifications. Homeostatic plasticity rules share the common feature that the history or actual state of global network activity influences the direction of neuroplastic changes induced by specific stimuli (Bienenstock et al., 1982; Abraham and Tate, 1997). Specifically, the effect of an actual stimulus is inversely correlated with the amount of background activity.

Homeostatic mechanisms are relevant in animal slice preparations and cultured neuronal networks (Li et al., 1998; Turrigiano et al., 1998) and at the system level in humans. In the human motor cortex, it has been shown that excitabilityenhancing fast repetitive transcranial magnetic stimulation (rTMS) increased the efficacy of a later excitability-diminishing slow rTMS protocol (Iyer et al., 2003). Moreover, enhancing or diminishing motor cortex excitability with transcranial direct

Received Dec. 12, 2006; revised March 4, 2007; accepted March 5, 2007.

This work was supported by the Bernstein Center for Computational Neuroscience (Goettingen, Germany). Correspondence should be addressed to Michael A. Nitsche at the above address. E-mail: mnitsch1@gwdg.de. D0I:10.1523/JNEUROSCI.5348-06.2007

Copyright $\odot 2007$ Society for Neuroscience $\quad$ 0270-6474/07/273807-06\$15.00/0 current stimulation (tDCS) resulted in inversely directed effects on excitability of identical rTMS protocols (Lang et al., 2004; Siebner et al., 2004). Because rTMS and tDCS induce widespread and not synapse-specific motor cortex excitability alterations, the results of these studies reflect a homeostatic regulation of background or general motor cortex excitability. However, the rules of homeostatic plasticity were originally dedicated to focal plastic changes of the strength of a limited amount of synapses relative to background activity, and these focal changes may resemble learning mechanisms more closely than global changes of excitability.

Paired associative stimulation (PAS) is an interesting tool to induce synapse-specific and thus learning-like neuroplastic modifications. It induces plasticity by simultaneous activation of intracortical neurons activated by motor cortex TMS and another population of neurons activated by somatosensory peripheral stimulation. With this selectivity, it may reflect the neurophysiological foundations of learning processes. A close relationship between motor learning and the amount of PAS-induced excitability-enhancing plasticity was demonstrated recently (Ziemann et al., 2004; Stefan et al., 2006).

We aimed to explore the impact of motor cortex background activity on associative plasticity. First (experiment 1), we identified PAS protocols resulting in short- or long-lasting excitability enhancements. We then evaluated the impact of a background activity enhancement by anodal tDCS or diminution by cathodal tDCS on subsequently (experiment 2) or simultaneously (experiment 3) applied PAS. Furthermore, we evaluated the effects of 
simultaneous tDCS and PAS for PAS protocol-eliciting longerlasting excitability enhancements (experiment 4), to study the dependency of homeostatic plasticity on the salience of plasticityinducing protocols. Because of the rules of homeostatic plasticity, excitability-diminishing cathodal tDCS should enhance, whereas excitability-enhancing anodal tDCS should reduce, the efficacy of PAS. By comparing different temporal combinations of the protocols, we aimed to explore the temporal characteristics of homeostatic plasticity. Applying salient and nonsalient PAS protocols for inducing long-lasting excitability changes, we tested the hypothesis that the quality of associative plasticity might determine whether homeostatic mechanisms apply.

\section{Materials and Methods}

Subjects. Six to 12 healthy subjects participated in each experiment. For experiments $1-3,12$ subjects were recruited (eight females; age, $25 \pm 2.5$ $\mathrm{SD}$ ). Six subjects participated in experiments 4 and 5 (five females; experiment 4: age, $25 \pm 3.9 \mathrm{SD}$; experiment 5: age, $28 \pm 1.9 \mathrm{SD}$ ). All subjects gave written informed consent. This study was approved by the ethics committee of the University of Goettingen, and we conform to the Declaration of Helsinki.

Measurement of motor cortical excitability. To monitor tDCS- and PASdriven changes of motor cortex excitability, muscle-evoked potentials (MEPs) of the right abductor digiti minimi muscle (ADM) were recorded after stimulation of their motor cortical representational fields by single-pulse TMS. These were induced using a Magstim 200 magnetic stimulator (Magstim Company, Dyfed, UK) and a figure-of-eight magnetic coil (diameter of one winding, $70 \mathrm{~mm}$; peak magnetic field, 2.2 tesla). The coil was held tangentially to the skull, with the handle pointing backwards and laterally at $45^{\circ}$ from midline. The optimum coil position was defined as the site where stimulation resulted consistently in the largest MEP. The surface EMG was recorded from the right ADM by use of $\mathrm{Ag}-\mathrm{AgCl}$ electrodes in a belly-tendon montage. The signals were amplified and filtered with a time constant of $10 \mathrm{~ms}$ and a low-pass filter of $2.5 \mathrm{kHz}$. Signals were then digitized at an analog-to-digital rate of $5 \mathrm{kHz}$ and relayed into a laboratory computer using the Signal 1.62 software (Cambridge Electronics Design, Cambridge, UK) and conventional averaging software. The intensity of the stimulator output was adjusted for baseline recording so that the average stimulus led to an MEP of $\sim 1 \mathrm{mV}$.

Direct current stimulation of the motor cortex. Direct currents were transferred by a pair of saline-soaked surface sponge electrodes and delivered by a specially developed, battery-driven, constant-current stimulator (Schneider Electronic, Gleichen, Germany) with a maximum output of $10 \mathrm{~mA}$. In each experiment, the motor-cortical electrode was fixed over the cortical representational field of the right ADM as identified by TMS and the other electrode contralaterally above the right orbit. The currents flowed continuously for $7 \mathrm{~min}$ before or during PAS in experiments 2,3 , and 5 to induce excitability shifts lasting for some minutes. In experiment 4, anodal or cathodal tDCS was administered for $15 \mathrm{~min}$. In former experiments, this stimulation duration generates after-effects lasting for $\sim 5-10 \mathrm{~min}$ ( $7 \mathrm{~min} \mathrm{tDCS}$ ) or $1 \mathrm{~h} \mathrm{(15} \mathrm{min} \mathrm{tDCS)} \mathrm{after} \mathrm{the} \mathrm{end}$ of stimulation (Nitsche and Paulus, 2001; Nitsche et al., 2003a). Motor cortex electrode size was $35 \mathrm{~cm}^{2}$ and current strength was $1 \mathrm{~mA}$ (current density, $\sim 0.03 \mathrm{~mA} / \mathrm{cm}^{2}$ ).

PAS. PAS was conducted according to the paradigm first described by Stefan et al. (2000). Only excitability-enhancing PAS protocols were used in this experiment. Peripheral nerve stimulation was performed on the right ulnar nerve at the wrist with an intensity $300 \%$ above sensory threshold with a Digitimer (Welwyn Garden City, UK) D185 stimulator. A single TMS pulse was delivered over the left motor cortical representation of the right ADM $25 \mathrm{~ms}$ after peripheral stimulation. TMS intensity was determined to elicit an MEP amplitude size of $\sim 1 \mathrm{mV}$. PAS was performed every $20 \mathrm{~s}$ for 7, 15, or $30 \mathrm{~min}$ in the different experiments.

Experimental procedures general experimental course. Each experiment was conducted in a repeated-measurement design. The order of the single sessions within an experimental protocol was randomized between subjects. A break of $\geq 1$ week was obligatory between single experimental sessions to avoid interference effects.
Subjects were seated in a reclining chair. After fixing the surface EMG electrodes above the right ADM, its left motor cortical representational field was identified by use of TMS (coil position that leads to the largest MEPs of ADM). Then, the TMS intensity resulting in MEP amplitudes of $\sim 1 \mathrm{mV}$ was established. For peripheral ulnar nerve stimulation, the optimum electrode position was identified and the electrode was fixed at this position with adhesive tape. Stimulation intensity was increased stepwise from a clear subthreshold level until the subjects felt a physical sensation. The lowest stimulation intensity that elicited a reliable sensation was defined as the threshold. Next, a baseline TMS measure was conducted to document pre-PAS motor cortex excitability. Twenty TMSs were recorded (frequency, $0.25 \mathrm{~Hz}$ ). Afterward, PAS/tDCS was performed. After PAS, TMS measures of excitability (baseline-intensity, 15 stimuli per time bin at $0.25 \mathrm{~Hz}$ ) were performed every 5 min for 30 min and then every $30 \mathrm{~min}$ for up to $90 \mathrm{~min}$ after stimulation. TMS intensity was held constant throughout the experiment.

Adjustment of PAS duration (experiment 1). This experiment was conducted to identify suitable PAS durations that would induce reliable short- or long-lasting excitability enhancements of the motor cortex. Because the original stimulation protocol (Stefan et al., 2000) lasts for 30 min and induces excitability changes stable for $>1 \mathrm{~h}$, it is not well suited to be combined with $\mathrm{tDCS}$. We therefore reduced PAS duration systematically from $30 \mathrm{~min}$, as described in the original protocol, to 15 and 7 min. After-effects were monitored up to 90 min after the end of PAS.

Modification of short-lasting PAS-induced excitability changes by previous administration of tDCS (experiment 2). This experiment was conducted for the 7 min PAS condition, which was shown to induce relatively short-lived, but still significant, excitability enhancements in experiment 1 . Anodal and cathodal tDCS were also administered for 7 min to induce short-lasting after-effects. Four experimental sessions were conducted for each subject: anodal tDCS followed by PAS or without PAS and cathodal tDCS followed by PAS or without PAS. DCS electrodes were fixed on the subject's head after identification of the left motor cortical ADM representation and baseline TMS measures, as described above. The PAS was administered immediately after the end of tDCS and removal of the respective electrodes. The after-effects of stimulation were measured as described above for $90 \mathrm{~min}$ after the end of intervention.

Modification of short-lasting PAS-induced excitability changes by simultaneous administration of $t D C S$ (experiment 3 ). The design of this experiment was identical to experiment 2, with the exception that anodal or cathodal tDCS was performed not before but during PAS in this condition. Consequently, TMS measures had to be performed through the tDCS electrodes, thus these were fixed after determination of the cortical ADM representation onto the head of the subjects and kept there for the whole course of the experiment. After-effects were measured for $90 \mathrm{~min}$ after intervention.

Modification of long-lasting PAS-induced excitability changes by simultaneous administration of tDCS (experiment 4). The protocol was identical to that of experiment 3, except for the fact that here 15 min PAS (which induces excitability enhancements stable for a longer duration than 7 min PAS) and 15 min anodal tDCS were combined. PAS was conducted with and without tDCS in different sessions separated by $\geq 1$ week. After-effects were monitored for $90 \mathrm{~min}$ after stimulation as described above and additionally on the evening of the stimulation day, as well as three times the day after stimulation (morning, noon, evening) for the combined PAS/tDCS protocol. TMS coil position, tDCS electrode position, and ADM recording electrode position were marked with a waterproof pen to guarantee constant electrode positions throughout the experiment.

Modification of tDCS-induced excitability changes by simultaneous TMS (experiment 5). In this control experiment, we tested whether simultaneous administration of tDCS and single-pulse TMS (20 s interstimulus interval) results in excitability changes comparable to those of tDCS with simultaneous PAS. The experimental protocol resembles experiment 3, anodal tDCS combined with PAS (and the respective tDCS-only measure), with the exception that the peripheral stimulus was omitted. Aftereffects were measured for up to 90 min after stimulation.

Calculations and statistics. MEP amplitude means were calculated for 
Table 1. Results of the ANOVAs conducted for the different experiments

\begin{tabular}{|c|c|c|c|}
\hline Experiments & $\mathrm{df}$ & Fvalue & $p$ value \\
\hline \multicolumn{4}{|l|}{ PAS only (experiment 1) } \\
\hline PAS & 2 & 27.487 & $<0.001^{*}$ \\
\hline Time course & 9 & 59.721 & $<0.001^{*}$ \\
\hline PAS $\times$ time course & 18 & 7.743 & $<0.001^{*}$ \\
\hline \multicolumn{4}{|l|}{ tDCS only } \\
\hline $\mathrm{tDCS}$ & 1 & 294.420 & $<0.001^{*}$ \\
\hline Time course & 9 & 0.885 & 0.568 \\
\hline $\mathrm{tDCS} \times$ time course & 9 & 43.447 & $<0.001^{*}$ \\
\hline \multicolumn{4}{|c|}{ Short-lasting PAS and tDCS (experiments 2,3) } \\
\hline Stimulation & 6 & 37.868 & $<0.001^{*}$ \\
\hline Time course & 9 & 24.187 & $<0.001^{*}$ \\
\hline Stimulation $\times$ time course & 54 & 14.043 & $<0.001^{*}$ \\
\hline \multicolumn{4}{|c|}{ Long-lasting PAS and tDCS (experiment 4) } \\
\hline Stimulation & 1 & 38.826 & $0.002^{*}$ \\
\hline Time course & 9 & 8.726 & $<0.001^{*}$ \\
\hline Stimulation $\times$ time course & 9 & 8.814 & $<0.001^{*}$ \\
\hline \multicolumn{4}{|c|}{ Single pulse TMS and tDCS (experiment 5) } \\
\hline Stimulation & 1 & 0.912 & 0.383 \\
\hline Time course & 9 & 19.994 & $<0.001^{*}$ \\
\hline Stimulation $\times$ time course & 9 & 0.990 & 0.462 \\
\hline
\end{tabular}

The asterisks mark significant main effects and interactions.

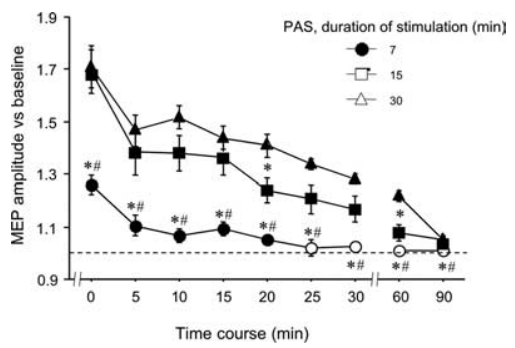

Figure 1. Dose-dependent effects of facilitatory PAS on motor cortex excitability. PAS duration was 7, 15, or $30 \mathrm{~min}$. The time course of baseline-standardized MEP amplitudes elicited by single-pulse TMS after PAS is depicted. Each protocol resulted in significant excitability enhancement compared with baseline (significant deviations from baseline, filled symbols) (paired two-tailed ttests; level of significance, 0.05 ). However, the 7 min PAS resulted in smaller and shorter-lasting excitability enhancements than 15 and 30 min PASs (significant differences between 7 and 15 min PASs, asterisks; between 7 and 30 min PASs, pound symbols) (paired two-tailed $t$ tests; level of significance, 0.05 ). Moreover, the 30 min PAS resulted in slightly larger excitability enhancements than the $15 \mathrm{~min}$ PAS (significant differences between 15 and 30 min PAS, asterisks) (paired two-tailed $t$ tests; level of significance, 0.05 ). The error bars indicate SEM. The dashed line indicates the baseline MEP amplitude.

each time bin covering the preintervention baseline (20 stimuli) and postintervention time points (15 stimuli). Postintervention MEP amplitude means were normalized to preintervention baselines. For experiment 1 , a repeated-measures ANOVA was calculated; the independent variables were the PAS duration and time course, and the dependent variable was the MEP amplitude. Student's $t$ tests (paired samples; twotailed; level of significance $<0.05$ ) were performed to determine whether the MEP amplitudes before and after PAS differed for each time bin and whether those differences depended on PAS duration.

Similarly, with regard to experiments 2, 3, 4, and 5, repeated-measure ANOVAs (independent variables, time course and stimulation condition; dependent variable, MEP amplitude) were calculated, then Student's $t$ tests (paired samples; two-tailed; level of significance $<0.05$ ) were performed to determine whether the MEP amplitudes before and after intervention differed for each time bin and whether those differences depended on the type of intervention. Additionally, baseline MEPamplitudes were controlled within each experiment for differences between interventions by Student's $t$ tests (paired samples; two-tailed; level of significance $<0.05$ ).

\section{Results \\ Adjustment of PAS duration (experiment 1)}

The results of the ANOVA display significant main effects of time and PAS protocol because of the fact that PAS induced significant enhancements of MEP amplitudes in all stimulation conditions and that these enhancements diminished over time (Table 1, Fig. 1). The significant interaction of PAS protocol and time course reflects the fact that prolonged PAS induced stronger and longer-lasting excitability enhancements than shorter protocols. Whereas 7 min PAS induced an initial increase in the MEP amplitude of $130 \%$ of baseline measures, which was diminished to a $10 \%$ increase after a few minutes and went back to baseline $25 \mathrm{~min}$ after the end of stimulation, 15 and $30 \mathrm{~min}$ PAS resulted in an MEP amplitude enhancement of $\sim 150 \%$ relative to baseline, which was significant up to $90 \mathrm{~min}$ after stimulation (Fig. 1). Baseline MEPs were identical in all conditions. Thus, the efficacy of PAS in inducing motor cortex excitability enhancements follows a dose-response relationship. Longer-lasting PAS induced increased effects compared with shorter protocols. For the PAS protocol, which includes $30 \mathrm{~min}$ stimulation, the results are comparable with those of former studies (Stefan et al., 2000).

\section{Modification of short-lasting PAS-induced excitability changes by previous or simultaneous administration of tDCS (experiments 2 and 3 )}

For the tDCS-only condition, the ANOVA reveals a significant main effect of tDCS and a significant interaction of $\mathrm{tDCS} \times$ time course (Table 1). This was caused by polarity-specific MEP increases or reductions observable for $\sim 60 \mathrm{~min}$ after the end of tDCS. Anodal tDCS increased, whereas cathodal tDCS diminished motor cortex excitability (Fig. 2). As shown by the results of the ANOVA conducted for all stimulation conditions, the main effects of stimulation and time course and the respective interaction between these factors were significant (Table 1). When excitability-enhancing anodal tDCS was applied before PAS, the resulting excitability enhancement was stronger and longer lasting compared with the application of PAS or tDCS alone. Cathodal tDCS administered before PAS, however, resulted in a slight diminution of the MEP amplitude after PAS and thus antagonized the effect of PAS alone (Fig. 3a). However, simultaneous application of PAS and tDCS resulted in an excitability diminution in the case of anodal tDCS, whereas simultaneous cathodal tDCS and PAS induced a prolonged excitability enhancement (Fig. 3b). Baseline MEPs were identical in all conditions. Seven minute anodal or cathodal tDCS alone resulted in motor cortex excitability enhancements and reductions similar in magnitude to former studies of our group (Nitsche and Paulus, 2001; Nitsche et al., 2003a). However, the respective effects were longer-lasting, most probably resulting from group specifics, because substantial interindividual variability of the proneness of subjects to react to tDCS was documented before (Nitsche and Paulus, 2001; Nitsche et al., 2003a, 2004a,b). 


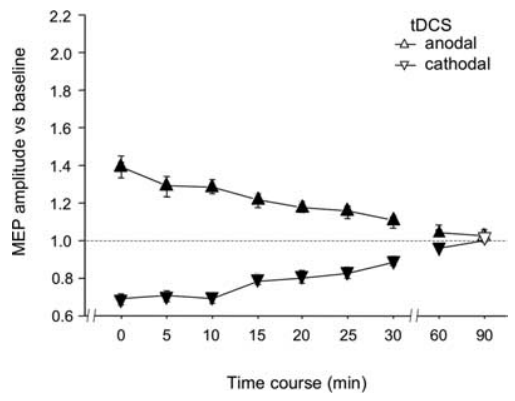

Figure 2. Effect of tDCS alone on motor cortex excitability. Anodal or cathodal tDCS was administered for $7 \mathrm{~min}$. The time course of baseline-standardized MEP amplitudes elicited by single-pulse TMS after TDCS is depicted. Anodal tDCS enhanced, whereas cathodal tDCS diminished, MEP amplitudes for $60 \mathrm{~min}$ relative to baseline. Filled symbols indicate significant differences between baselines and post-tDCS MEPs (paired two-tailed $t$ tests; level of significance, 0.05). Error bars indicate SEM. The dashed line indicates the baseline MEP amplitude.

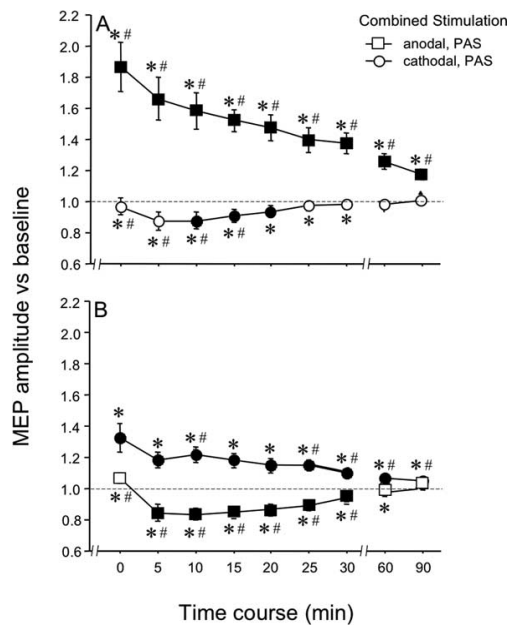

Figure 3. Effect of combined tDCS and PAS on motor cortex excitability. The time course of baseline-standardized MEP amplitudes elicited by single-pulse TMS after PAS combined with tDCS (20 PASs; 7 min tDCS) is depicted. $A$, When tDCS was applied before PAS, combined anodal tDCS and PAS resulted in an excitability enhancement exceeding in magnitude and duration than the excitability change elicited by anodal tDCS or PAS alone, whereas cathodal tDCS combined with PAS resulted in a minor excitability reduction that, however, was smaller than the excitability diminution achieved by cathodal tDCS alone. $\boldsymbol{B}$, When PAS and tDCS were administered simultaneously, anodal tDCS and PAS resulted in a prolonged excitability diminution, whereas cathodal tDCS applied together with PAS enhanced excitability. Filled symbols indicate significant deviations of the postintervention MEP amplitudes relative to baseline MEPs, and asterisks indicate significant differences to tDCS of the same polarity given alone and pound symbols to PAS alone (paired two-tailed $t$ tests; level of significance, 0.05 ). Error bars indicate SEM. The dashed line indicates the baseline MEP amplitude.

\section{Modification of long-lasting PAS-induced excitability changes by simultaneous administration of $\mathrm{tDCS}$ (experiment 4)}

The results of the respective ANOVA display significant main effects of time course and stimulation as well as a significant interaction between these factors (Table 1). This is because of a significant excitability enhancement induced by 15 min PAS, which, however, is turned into inhibition if combined with simultaneous anodal tDCS (Fig. 4). Interestingly, this diminution of MEP amplitudes remains until the noon after the stimulation day and thus for $\sim 24 \mathrm{~h}$ after stimulation. Because prolonged tDCS and PAS resulted in identically directed homeostatic plasticity changes, compared with the shorter-lasting protocols, the stability of the neuroplastic excitability changes seems not to be

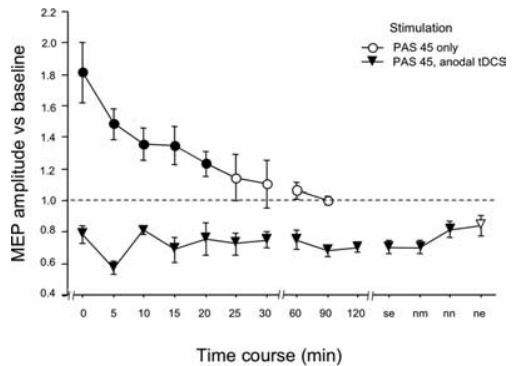

Figure 4. Prolonged tDCS/PAS enhances the duration of homeostatic plasticity. The time course of baseline-standardized MEP amplitudes elicited by single-pulse TMS after PAS combined with $\mathrm{tDCS}$ (45 PASs; 15 min anodal tDCS) is depicted. Simultaneously administered anodal tDCS converts the excitability-enhancing effect of PAS into inhibition. This effect is directed identically to the shorter stimulation protocol, but the excitability diminution is significantly prolonged up to midday after the day of stimulation. Filled symbols indicate significant differences relative to baseline MEPs. Error bars indicate SEM. The dashed line indicates the baseline MEP amplitude. se, Same evening; nm, next morning; nn, next noon; ne, next evening.

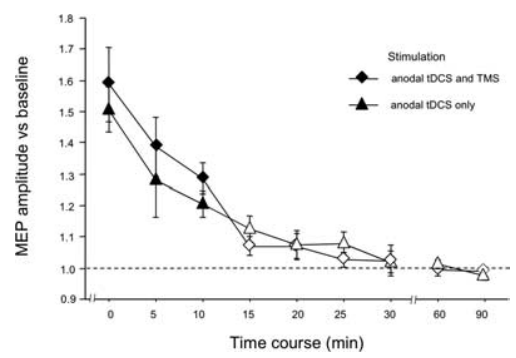

Figure 5. TMS alone does not induce homeostatic alterations of anodal tDCS-elicited plasticity. The time course of baseline-standardized MEP amplitudes elicited by single-pulse TMS after anodal tDCS only for $7 \mathrm{~min}$ and anodal tDCS combined with TMSs (every $20 \mathrm{~s}$, thus resembling the TMS frequency of the respective PAS protocol) is depicted. Both protocols resulted in identical excitability enhancements. Filled symbols indicate significant differences relative to baseline MEPs. Error bars indicate SEM. The dashed line indicates the baseline MEP amplitude.

critical. Baseline MEP amplitudes did not differ between both stimulation conditions.

\section{Modification of tDCS-induced excitability changes by simultaneous TMS (experiment 5)}

Here, the ANOVA reveals a significant main effect of time course, but the main effect of stimulation as well as the interaction between both variables turned out to be not significant (Table 1). This is because of an identical MEP amplitude enhancement accomplished by anodal tDCS ( $7 \mathrm{~min}$ ) alone and the combination of tDCS with TMS (single pulse every $20 \mathrm{~s}$ ), which is significant for up to $10 \mathrm{~min}$ after the end of stimulation (Fig. 5). Baseline MEP amplitudes are identical for both stimulation conditions.

Thus, TMS alone does not suffice to induce homeostatic plasticity when administered simultaneously with tDCS. We conclude that the homeostatic effect observed after simultaneous tDCS/PAS is indeed caused by an influence of tDCS on associative synapse-specific plasticity, as induced by PAS.

\section{Discussion}

Homeostatic plasticity (i.e., the dependency of the amount and direction of the obtainable plasticity from the history of activity of a neuronal network) is increasingly recognized as regulatory mechanism for keeping neuronal modifications within a reasonable physiological range. Here, we demonstrate an acute homeostatic effect of global network level of activity on synapse-specific 
plasticity, which only occurs if both plasticity-inducing protocols are applied simultaneously.

PAS, which induces a motor cortex excitability enhancement when applied alone, resulted in an excitability diminution when combined simultaneously with excitability-enhancing tDCS. If excitability-reducing tDCS was coadministered, it increased and prolonged the PAS-induced excitability enhancement. Control experiments revealed that (1) this effect indeed depends on the interaction of tDCS and PAS, because administering solely the TMS stimulus of the PAS protocol did not influence tDCSinduced plasticity, and (2) this homeostatic effect is not restricted to plasticity protocols inducing short-lasting plasticity, because prolonged tDCS and PAS protocols resulted in identically directed effects. Conversely, tDCS applied before PAS did modify the PAS-induced excitability changes synergistically. Thus, the effect of background activity/excitability on associative plasticity critically depends on their temporal relationship: whereas previous background activity modulates associative plasticity synergistically, simultaneous modification of background and associative, synapse-specific plasticity modifies the latter antagonistically and thus in a homeostatic manner.

Traditionally, homeostatic plasticity is thought to act within an extended time scale. In animal experiments, enhanced or reduced basal neuronal network activity held constant for days or weeks influences the effects of acute plasticity-inducing protocols homeostatically (Burrone and Murthy, 2003; Turrigiano and Nelson, 2004). However, homeostatic mechanisms can work on a much shorter time scale in animals (van Welie et al., 2004) and humans (Iyer et al., 2003; Lang et al. 2004; Siebner et al., 2004). In humans, preconditioning of the motor cortex with excitabilitymodifying external stimulation (rTMS or tDCS) determines the direction of neuroplastic excitability changes induced by a succeeding stimulation protocol homeostatically. In contrast to these studies, in which stimulation protocols were applied that involve the whole cortical area stimulated, the current experiments probed the effect of a global network plasticity change on synaptically restricted associative plasticity. Our results differ clearly from those of the former studies: preconditioning of associative plasticity with global excitability-enhancing tDCS boosted the efficacy of the subsequent PAS protocol to produce excitatory neuroplasticity, whereas excitability-diminishing tDCS reduced it. However, simultaneous global network modification and associative plasticity induction reversed these effects (i.e., induced homeostatic plasticity).

The differential effects of preconditioning versus simultaneous network modification on associative plasticity give rise to two main questions: why does this kind of homeostatic plasticity follow a different time rule than homeostatic mechanisms induced by other stimulation protocols, and what is the underlying physiological mechanism? In recent studies in humans, consecutively administered neuroplasticity-inducing stimulation protocols induced homeostatic effects (Iyer et al., 2003; Lang et al., 2004; Siebner et al., 2004). In two of these studies, the preconditioning stimulation protocol was identical to the current one (i.e., tDCS). The main difference lies in the conditioning protocol, which was rTMS in the former studies but PAS in the current one. PAS induces timing-dependent, synapse-specific neuroplasticity. rTMS, in contrast, induces neuroplasticity, which may not be restricted to specific synaptic connections. In doing so, rTMS might induce patterns of neuroplasticity similar to that of tDCS. Thus, subsequent neuroplasticity-inducing protocols that have similar effects on global network plasticity may work antagonis- tically to keep global network function within a physiological range.

However, synapse-specific and thus spatially restricted neuroplasticity, as induced by PAS, might not suffice to induce counteracting homeostatic mechanisms. It thus might profit from previous global excitability-enhancing neuroplasticity and be diminished by antecedent global excitability-diminishing neuroplasticity. However, simultaneous administration of tDCS and PAS did induce homeostatic plasticity. Such a homeostatic mechanism makes sense for optimizing information processing, because it would strengthen synapse-specific plasticity in a silent network while suppressing it during high-level general network activity. So what is the physiological difference between succeeding and simultaneous $\mathrm{tDCS} / \mathrm{PAS}$ protocols causing these dissociating effects? Although knowledge about the mechanisms responsible for the induction of neuroplasticity by tDCS and PAS is limited, it is known that ion channels are involved in the induction of plasticity and NMDA receptors in the stabilization of the after-effects of both plasticity-inducing stimulation protocols (Liebetanz et al., 2002; Stefan et al., 2002; Nitsche et al., 2003b). Because it has been shown that intraneuronal calcium concentration regulates homeostatic plasticity (Misonou et al., 2004), this could be a candidate mechanism. However, alternative processes cannot be ruled out by the results of our experiments. Thus, the specific mechanisms of action have to be explored in future studies.

Associative plasticity is thought to be a neurophysiological correlate of learning and memory formation in humans and animals (Rioult-Pedotti et al., 1998, 2000; Ziemann et al., 2004; Stefan et al., 2006). Recently, motor cortex tDCS has been demonstrated to improve motor and visuomotor coordination learning in humans (Nitsche et al., 2003c; Antal et al., 2004). Given the results of our study, an optimum effect on boosting learningrelated associative plasticity should be achieved by excitabilityenhancing anodal tDCS administered before the actual learning process or excitability-reducing cathodal tDCS during learning. However, if applied during learning only anodal but not cathodal tDCS improved learning substantially in both of the abovementioned studies. Furthermore, if applied before the behavioral task, anodal and cathodal tDCS did not modify performance in one of the paradigms and had only minor effects in the other (unpublished results of our group). These results are in clear opposition to the effects of tDCS on PAS. One explanation might be that PAS indeed resembles one important synaptic derivate of learning processes, but the frequency of application of associative stimuli is much too low to realistically represent neuronal activity during complex learning processes, as tested in the abovementioned studies. In this way, PAS might represent an activity insufficient on its own to serve as a salient signal against increased actual background activity and thus might be toned down. This argument does not rule out that PAS resembles neuronal derivates of learning and memory formation in principle. The studies of Ziemann et al. (2004) and Stefan et al. (2006) have demonstrated elegantly its involvement, but the results of our study put into question whether the specific low-frequency PAS protocol applied here is sufficient to mimic learning processes. Alternatively, it might be argued that homeostatic effects of tDCS on motor learning and PAS-induced plasticity dissociate on a time scale: in another study of our group (Lang et al., 2003), it was shown that anodal tDCS administered during motor learning selectively impaired recall of the learned sequence. This result might argue for a delayed homeostatic effect of background excitability enhancement on memory consolidation. Additional 
studies are needed to clarify the specific relationship of PAS and learning to a greater extent.

In a recent study, Baumgartner et al. (2007) have demonstrated a relevant impact of emotional state and arousal on the size of MEP amplitudes. We think that we can exclude a relevant influence of these parameters or the results of our study for the following reasons. First, in a recent study, tDCS of the dorsolateral prefrontal cortex, which modifies mood in depressed subjects, did not influence emotional state and arousal in healthy subjects (our unpublished observations). Second, modulation of general arousal or emotional state during the course of the experiment would result in similar MEP enhancements independent of the stimulation protocol used. On the contrary, our results strongly depend on the specific stimulation protocol. Moreover, because we used a randomized repeated-measurement design, any change of arousal or emotional state caused by, for example, the first participation of one subject in this kind of experiment would be randomly assigned to the specific experimental conditions, and thus its systematic contribution to the results is improbable. However, the results of the study of Baumgartner et al. (2007) point to the general fact that it is important to control for emotional state and arousal during the conduction of experiments using MEPs as dependent variables.

Together, the results of our study present a new homeostatic plasticity mechanism, which only applies when associative plasticity is induced during enhanced or reduced background activity. This kind of homeostatic plasticity thus clearly differs from other metaplastic mechanisms described so far and sheds light on the fact that multiple homeostatic mechanisms might exist that differ because of the kinds of plasticity induced as well as because of the time course of plasticity induction. Future studies should focus on the underlying mechanisms of these different kinds of metaplasticity and explore their functional relevance.

\section{References}

Abraham WC, Tate WP (1997) Metaplasticity: a new vista across the field of synaptic plasticity. Prog Neurobiol 52:303-323.

Antal A, Nitsche MA, Kinsces TZ, Kruse W, Hoffmann K-P, Paulus W (2004) Facilitation of visuo-motor learning by transcranial direct current stimulation of the motor and extrastriate visual areas in humans. Eur J Neurosci 19:2888-2892.

Baumgartner T, Willi M, Jancke L (2007) Modulation of corticospinal activity by strong emotions evoked by pictures and classical music: a transcranial magnetic stimulation study. NeuroReport 18:261-265.

Bienenstock EL, Cooper LN, Munro PW (1982) Theory for the development of neuron selectivity: orientation specificity and binocular interaction in visual cortex. J Neurosci 2:32-48.

Burrone J, Murthy VN (2003) Synaptic gain control and homeostasis. Curr Opin Neurobiol 13:560-567.

Iyer MB, Schleper N, Wassermann EM (2003) Priming stimulation enhances the depressant effect of low-frequency repetitive transcranial magnetic stimulation. J Neurosci 23:10867-10872.

Lang N, Nitsche MA, Sommer M, Tergau F, Paulus W (2003) Modulation of motor consolidation by external DC stimulation. Suppl Clin Neurophysiol 56:277-281.

Lang N, Siebner HR, Ernst D, Nitsche MA, Paulus W, Lemon RN, Rothwell JC (2004) Preconditioning with transcranial direct current stimulation sensitizes the motor cortex to rapid-rate transcranial magnetic stimulation and controls the direction of after-effects. Biol Psychiatry 56:634-639.
Li H, Weiss SR, Chuang DM, Post RM, Rogawski MA (1998) Bidirectional synaptic plasticity in the rat basolateral amygdala: characterization of an activity-dependent switch sensitive to the presynaptic metabotropic glutamate receptor antagonist $2 S \alpha$-ethylglutamic acid. J Neurosci 18:1662-1670.

Liebetanz D, Nitsche MA, Tergau F, Paulus W (2002) Pharmacological approach to synaptic and membrane mechanisms of DC-induced neuroplasticity in man. Brain 125:2238-2247.

Misonou H, Mohapatra DP, Park EW, Leung V, Zhen D, Misonou K, Anderson AE, Trimmer JS (2004) Regulation of ion channel localization and phosphorylation by neuronal activity. Nat Neurosci 7:711-718.

Nitsche MA, Paulus W (2001) Sustained excitability elevations induced by transcranial DC motor cortex stimulation in humans. Neurology 57:1899-1901.

Nitsche MA, Nitsche MS, Klein CC, Tergau F, Rothwell JC, Paulus W (2003a) Level of action of cathodal DC polarisation induced inhibition of the human motor cortex. Clin Neurophysiol 114:600-604.

Nitsche MA, Fricke K, Henschke U, Schlitterlau A, Liebetanz D, Lang N, Henning S, Tergau F, Paulus W (2003b) Pharmacological modulation of cortical excitability shifts induced by transcranial DC stimulation. J Physiol (Lond) 553:293-301.

Nitsche MA, Schauenburg A, Lang N, Liebetanz D, Exner C, Paulus W, Tergau F (2003c) Facilitation of implicit motor learning by weak transcranial direct current stimulation of the primary motor cortex in the human. J Cog Neurosci 15:619-626.

Nitsche MA, Grundey J, Liebetanz D, Lang N, Tergau F, Paulus W (2004a) Catecholaminergic consolidation of motor cortex plasticity in humans. Cereb Cortex 14:1240-1245.

Nitsche MA, Jaussi W, Liebetanz D, Lang N, Tergau F, Paulus W (2004b) Consolidation of externally induced human motor cortical neuroplasticity by d-cycloserine. Neuropsychopharmacology 29:1573-1578.

Rioult-Pedotti MS, Friedman D, Hess G, Donoghue JP (1998) Strengthening of horizontal cortical connections following skill learning. Nat Neurosci 1:230-234.

Rioult-Pedotti MS, Friedman D, Hess G, Donoghue JP (2000) Learninginduced LTP in neocortex. Science 290:533-536.

Siebner HR, Lang N, Rizzo V, Nitsche MA, Paulus W, Lemon RN, Rothwell JC (2004) Preconditioning of low-frequency repetitive transcranial magnetic stimulation with transcranial direct current stimulation: evidence for homeostatic plasticity in the human motor cortex. J Neurosci 24:3379-3385.

Stefan K, Kunesch E, Cohen LG, Benecke R, Classen J (2000) Induction of plasticity in the human motor cortex by paired associative stimulation. Brain 123:572-584.

Stefan K, Kunesch E, Benecke R, Cohen LG, Classen J (2002) Mechanisms of enhancement of human motor cortex excitability induced by interventional paired associative stimulation. J Physiol (Lond) 543:699-708.

Stefan K, Wycislo M, Gentner R, Schramm A, Naumann M, Reiners K, Classen J (2006) Temporary occlusion of associative motor cortical plasticity by prior dynamic motor training. Cereb Cortex 16:376-385.

Turrigiano GG, Nelson SB (2004) Homeostatic plasticity in the developing nervous system. Nat Rev Neurosci 5:97-107.

Turrigiano GG, Leslie KR, Desai NS, Rutherford LC, Nelson SB (1998) Activity-dependent scaling of quantal amplitude in neocortical neurons. Nature 391:892-896.

van Welie I, van Hooft JA, Wadman WJ (2004) Homeostatic scaling of neuronal excitability by synaptic modulation of somatic hyperpolarizationactivated Ich channels. Proc Natl Acad Sci USA 101:5123-5128.

Ziemann U, Ilic TV, Pauli C, Meintzschel F, Ruge D (2004) Learning modifies subsequent induction of long-term potentiation-like and long-term depression-like plasticity in human motor cortex. J Neurosci 24:16661672. 